\title{
CONSOANTES EM FINAL DE PALAVRA EM PROTO-PÁNO
}

\section{FINAL CONSONANTS IN PROTO-PÁNO}

\author{
Sanderson Soares Castro de Oliveira \\ Universidade do Estado do Amazonas, UEA, Tabatinga, AM, Brasil
}

Resumo: O presente artigo é uma versão levemente modificada do capítulo 4 da tese Contribuiçóes para a Reconstrução do Protopáno, orientada parcialmente pelo professor Aryon Dall'Igna Rodrigues, entre os anos de 2009 e 2012, e defendida sob a orientaçáo da professora Ana Suelly Arruda Câmara Cabral. A tese é uma revisão da reconstrução do Protopáno apresentada em Shell (1975 [1965]), e o capítulo fundamenta: a) a existência de consoantes oclusivas (incluindo $n$, [Loos, 1978]) em línguas do subgrupo Mayorúna; b) a existência de uma alternância entre formas longas e formas curtas, relacionadas respectivamente aos casos ergativo e absolutivo; c) a existência de consoantes oclusivas em margem direita de sílaba em Protopáno.

Palavras-chave: Família Páno; Protopáno; Padrão Silábico em Páno; Linguística Histórica

Abstract. The present article is a slightly modified version of chapter 4 of the doctoral dissertation Contribuiçôes para a Reconstrução do Protopáno, initially supervised by Aryon Dall'Igna Rodrigues, between 2009-2012, and finalized under the orientation of Ana Suelly Arruda Câmara Cabral. The thesis is a revision of Proto-Páno reconstruction by Shell(1975 [1965]), and the chapter demonstrates: a) the existence of oclusive consonants (including $n$, [cf. Loos, 1978]) in languages of the Mayorúna subgroup; b) the existence of an alternation involving long and short forms, related respectivelly to the ergative and absolutive case in all Panoan languages; c) the existence in Proto-Páno of oclusive consoants in coda position.

Keywords: Panoan Family; Proto-páno; Sylabic Patterns in ProtoPáno; Historical Linguistics 


\section{Introduçáo}

No presente artigo, discutimos a reconstruçáo de consoantes oclusivas ${ }^{1}$ em margem direita de sílaba final de palavra em Protopáno ${ }^{2}$. Presentemente, os dados disponíveis fundamentam a reconstrução de consoantes finais nessa posição da palavra. Há línguas como Korúbo, Matís e Mayorúna que apresentam as consoantes oclusivas $t, d, k$ e $n$ em margem direita de sílaba no interior e no final de palavra. Entretanto, por precaução, decidimos não considerar esses dados como fundamentos para a reconstruçáo de consoantes oclusivas nessa última posição, mas não excluímos a possibilidade de que, em algum estágio anterior da família Páno, consoantes oclusivas seguissem as mesmas orientações fonotáticas das demais consoantes. Pelas razóes expostas, restringimo-nos, neste estudo, a reconstruir consoantes oclusivas em margem direita de sílaba final de palavra, para o que consideramos as fortes evidências disponíveis em línguas de diferentes sub-ramos da família.

O artigo está assim organizado: na seção 2, fazemos algumas consideraçôes sobre a análise de Shell (1975 [1965]) para as formas trissilábicas da família Páno e sobre as observações de Girard (1971) acerca desse tópico; na seção 3, discutimos a existência de formas longas e curtas na família Páno e propomos uma definição para esses termos que foram usados até aqui de uma forma bastante livre; na seção 3.1, apresentamos a nossa proposta de reconstruçáo de consoantes finais para a protolíngua e as evidências que fundamentam essa hipótese; em 3.2, discutimos a natureza sonora dos segmentos que teriam ocorrido em posição final de palavra; e, em 4, discutimos brevemente os reflexos dessas consoantes em Kaxararí.

\section{Formas trissilábicas reconstruídas por Shell (1975 [1965])}

Segundo Shell (1975 [1965], p. 93), existiria em Protopáno nomes e verbos monomorfêmicos monossilábicos, bissilábicos e trissilábicos. No caso de palavras trissilábicas, os nomes e os verbos teriam tido desenvolvimentos distintos nas diversas línguas. Interessa-nos, neste artigo, principalmente, as

\footnotetext{
${ }^{1}$ A definição de consoantes oclusivas aqui apresentada inclui consoantes nasais e baseia-se no artigo de Loos (1978).

${ }^{2}$ Muito embora a discussão esteja centrada na sílaba em final de palavras, o reconhecimento do novo padrão silábico teria consequências também para a sílaba no interior da palavra, principalmente, em palavras polimorfêmicas. Essa questấo merece um aprofundamento maior. Ademais, há que se reconhecer ainda que as línguas da família Páno e, consequentemente, o Protopáno também possuem outras consoantes em coda, mas que não estão consideradas por não serem diretamente relacionadas com a discussão aqui apresentada.
} 
formas nominais trissilábicas reconstruídas por Shell (1975 [1965], p. 93) para a protolíngua e seus reflexos nas línguas contemporâneas.

A terceira sílaba das formas nominais reconstruídas por Shell (1975 [1965]) é, talvez, a única característica do "Páno-Reconstruído" que, segundo a autora, apresenta relação com todos os níveis linguísticos analisados por ela e a sua reconstruçáo em nomes teria implicaçôes para a fonologia segmental, suprassegmental e mesmo para o "traço de referência transitiva". A terceira sílaba Páno, proposta por Shell, tem influenciado várias análises de diferentes aspectos de línguas da família (COSTA, 2000; VALENZUELA, 2003; CÓRDOBA; VALENZUELA; VILLAR, 2011).

O principal fundamento para a reconstrução de uma terceira sílaba Páno por Shell (1975 [1965]) é o "conservantismo e resistência à redução silábica” em Chákobo, conforme observado por Girard (1971, p. 150). Esse "conservantismo" consiste na existência, em Chákobo, de uma sílaba a mais em palavras que, em outras, seria inexistente, como podemos ver nas seguintes reconstruçóes de Shell (1975 [1965]): 
Tabela 11 - Formas trissilábicas, segundo Shell (1975)

\begin{tabular}{|c|c|c|c|c|}
\hline & Protopáno & Ksh & SK & $\mathrm{Cp}$ \\
\hline 3. & *2amĩno & Pa'mí & Pa'mi; & Pa'min; \\
\hline 8. & *2awara & $\mathrm{PO}^{\prime} \mathrm{O}$ & Pa'wa & Pa'wa \\
\hline 9. & *?awini & -- & $a^{\prime}=\tilde{1}$ & $a^{\prime}$ win \\
\hline 14. & *2iani & Piãi & Piãi & Pián \\
\hline 15. & *2i?saka & Pisá & Pisá & Piisá \\
\hline 17. & *?itsisa & Pitsís & -- & -- \\
\hline 20. & *?inak & -- & Piná & Pinaa \\
\hline 32. & *[?]o?possi & -- & hopós & Po?pós \\
\hline 50. & *ãs[t]ini & asi & hasi & hasín \\
\hline 57. & * Ba?kifi & ßakíf- & Bakís & Ba?kís \\
\hline 74. & 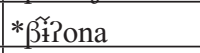 & $\beta$ ßiõ & $\beta$ iัõ & -- \\
\hline 86. & *ßitimi & $\beta \mathrm{it} \tilde{\mathrm{ti}}$ & $\beta i \tilde{\mathrm{t}} \tilde{\mathrm{i}}$ & -- \\
\hline 103. & *ßokono & Bokõ & Bokó & ßokón \\
\hline 165. & *kamano & kamõ & kamãi & - \\
\hline 168. & *kanapa & Kaná & kaná & kaná \\
\hline 171. & *kapiti & kapi & kapii & kapii \\
\hline 234. & *mawisi & Maís & mawís & -- \\
\hline 239. & *mirtsis[t]i & -- & mitsis & mintsís \\
\hline 240. & *mikini & mikî & mikr & mikin \\
\hline 253. & *naipa & Naí & naí & naí \\
\hline 255. & *nak ${ }^{\text {wasi }}$ & nak $^{w} \tilde{a} s$ & nakãs & nakás \\
\hline 293. & *nonoma & nonõ & nonõ & nonõ \\
\hline 327. & *piraka & Piaka & piá & pi?asa \\
\hline 348. & *poyami & piã & poyã & poyán \\
\hline 352. & *raßita & $\mathrm{ra} \beta \mathrm{i}$ & $\mathrm{ra} \beta \dot{\mathrm{i}}$ & $\mathrm{ra} \beta \dot{\mathrm{i}}$ \\
\hline 395. & *sakata & șaká & șaká & saka \\
\hline 401. & *satano & -- & -- & -- \\
\hline 402. & *sawani & Şว & sawã & sawán \\
\hline 475. & *[w]asnona & -- & wanõ & -- \\
\hline 476. & *wasmini & -- & wasmî & wasmin \\
\hline 491. & *ya?nani & naĩ & yaã & ya?nán \\
\hline 499. & *yawis $\int i$ & naĩ & yawís & yawís \\
\hline 503. & *yoßika & -- & уо $\beta і$ & yo $\beta$ i \\
\hline 511. & *yofini & $\mathrm{u} \int_{\tilde{1}}$ & yo $\int \tilde{1}$ & yofín \\
\hline
\end{tabular}




\begin{tabular}{|c|c|c|c|c|}
\hline & $\mathrm{Ch}$ & $\mathrm{A}$ & $\mathrm{Kn}$ & $M$ \\
\hline 3. & Pámino & $\mathrm{am} \tilde{i}$ & $\mathrm{am} \tilde{\mathrm{i}}$ & $\mathrm{am} \tilde{i}$ \\
\hline 8. & Páwara & áá & awa & áwa \\
\hline 9. & Pa'wini & \begin{tabular}{|l|l|}
$a \tilde{\imath}$ \\
\end{tabular} & ã $\mathbf{1}$ & 'ãwĩ \\
\hline 14. & Piáni & Ĩyã & ĩã & $\tilde{\mathrm{i} a ̃ a}$ \\
\hline 15. & Pisaka & isaa & isa & - \\
\hline 17. & Pitsisa & itsis & -- & -- \\
\hline 20. & Pinaka & iná & iná & -- \\
\hline 32. & hopiși & hõpox & hũpus & íposi \\
\hline 50. & hasíni & hãsĩ & hasĩ & ásĩ \\
\hline 57. & bakísi & -- & bakis & фákifi \\
\hline 74. & Biłóna & 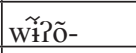 & -- & $\phi \dot{1} \tilde{O}$ \\
\hline 86. & $\beta$ itiimi & witi & bit $\widetilde{t}$ & $\phi \dot{i i t \tilde{t}}$ \\
\hline 103. & Bokóno & wokõ & buku & фókõ \\
\hline 165. & kamáno & Sinokamã & kamã & kámã \\
\hline 168. & kanápa & kanáa & kana & kanã \\
\hline 171. & kápiti & kapii & kapì & kápĩ \\
\hline 234. & -- & mais & mais & máwisi \\
\hline 239. & mitsisi & mîtsis & mîtsis & mîtsisi \\
\hline 240. & mikini & mikî & mik $\widetilde{i}$ & miki \\
\hline 253. & naipa & naí & nai & nai \\
\hline 255. & -- & nakax & nakas & nákași \\
\hline 293. & nonóma & nõnõ & nũnũ & nõnõ \\
\hline 327. & piráka & piraa & -- & pía \\
\hline 348. & poyámi & põyã & pũyã & põyã \\
\hline 352. & ráßita & rawii & dabi & rá $\phi \dot{i}$ \\
\hline 395. & sakáta & xakaa & saka & şakã \\
\hline 401. & satáno & xatã & -- & sáta \\
\hline 402. & sawáni & xãã & Sawã & sãwã \\
\hline 475. & Basnona & wasnõ & basnũ & -- \\
\hline 476. & wasmini & -- & -- & -- \\
\hline 491. & ya?áni & yanã & yanã & yánã \\
\hline 499. & -- & yais & yaif & yáwijí \\
\hline 503. & yóßika & yowii & -- & yó \\
\hline 511. & yođíni & yo $\int \tilde{1}$ & $\mathrm{yu} \int \tilde{1}$ & yó $\int \tilde{1}$ \\
\hline
\end{tabular}


Além de considerar os reflexos em Chákobo, Shell (1975 [1965]) também considera o acento na última sílaba à direita de palavras bissilábicas das línguas Kashíbo, Shípibo-Kónibo e Kapanáwa, correspondendo à penúltima sílaba das palavras de trissilábicas Chákobo, como indicaçôes de que teria existido uma terceira sílaba nessas línguas. Ela observa que o acento nessas três línguas ocorre, geralmente, na sílaba mais à esquerda em palavras bissilábicas, mas, nos reflexos acima, ocorre sempre na sílaba mais à direita.

Nos casos em que, nas três línguas, além do acento, há reflexo nasal na última vogal, Shell (1975 [1965]) considerou que a consoante da sílaba final teria sido ${ }^{*} n$ ou ${ }^{*} m$, representando esta sílaba como $n / m V$. 
Tabela 12 - Palavras trissilábicas reconstruídas por Shell (1975), cuja terceira sílaba terminava em consoante nasal, mas sem especificaçáo da realização fonética da sílaba na protolíngua

\begin{tabular}{|c|c|c|c|c|c|c|c|c|}
\hline & Protopáno & Ksh & SK & $\mathrm{Cp}$ & $\mathrm{Ch}$ & $\mathrm{A}$ & $\mathrm{Kn}$ & $\mathrm{M}$ \\
\hline 29. & *?ijmi[n?mV] & Pifmi & Pi $\int \mathrm{mi}$ & Pifmín & -- & $\mathrm{i} \int \mathrm{mi}$ & ifmĩ & ifmĩ \\
\hline 69. & *ßawi[n?mV] & $\beta a i$ & ßawi & Bawín & -- & wãi & bãi & фãwĩ \\
\hline 75. & * $\beta \mathrm{it} \int \mathrm{o}[\mathrm{nPmV}]$ & $\beta$ itsõ & $\beta$ ittsõi & $\beta$ itfo & -- & 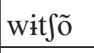 & bitfu))- & ini $\phi$ itfõi \\
\hline 79. & * $\beta$ iрo $[\mathrm{n} 2 \mathrm{mV}]$ & $\beta$ іipĩ & $\beta$ їро & Bipón & -- & wipõ & bĩpu & $\phi$ ipo \\
\hline 85. & *ßișna[n?mV] & Bișnã & Bişnãi & Bișnán & -- & wixna & bișnã & фìișnã \\
\hline 94. & * $\beta$ ino $[\mathrm{n} ? \mathrm{mV}]$ & $\beta$ inõ & Binó & -- & -- & winõ & -- & -- \\
\hline 108. & *ßõsi[n?mV] & $\beta$ õsime & $\beta$ õsi & hini $\beta$ osi & -- & wõsi & -- & фósi \\
\hline 200. & *kõsa[n?mV] & kõșã & kõşá & kõşá & -- & koxã & kuşa & koşa \\
\hline 204. & *ma?i[n?mV] & maĩ & maĩ & -- & -- & mã? & mai & mãĩ \\
\hline 207. & *mãt & -- & mãt & mant $\int a ́ n$ & -- & mat $\int a ̃$ & -- & -- \\
\hline 212. & *maka[n?mV] & -- & maká & mánkan & -- & makã & mãkã & makantJiu \\
\hline 225. & $*_{\mathrm{ma}}[\mathrm{s}] \mathrm{i}[\mathrm{n} ? \mathrm{mV}]$ & masĩ & masĩ & masín & -- & mãsĩ & -- & máşị \\
\hline 252. & *na?i[n?mV] & -- & naĩ & naiín & -- & nã? & nãi & nãĩ \\
\hline 286. & *no?i[n?mV] & noĩ & -- & no?ín & -- & nõ? & nui & nõi \\
\hline 335. & *piSi[n?mV] & pisí & pi $\int \tilde{1}$ & 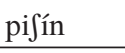 & -- & pifĩ & pi $\int \mathrm{i}$ & pí $\int \tilde{1}$ \\
\hline 344. & $* \mathrm{p}[\tilde{\mathrm{o}}] \mathrm{si}[\mathrm{nPmV}]$ & pos̊ri & põ $\tilde{S}$ & -- & -- & posi & -- & póşî̀ \\
\hline 379. & *sani[n?mV] & sanĩ & -- & -- & -- & sanĩ & sanĩ & sánĩ \\
\hline 385. & * fiko[n?mV] & Sîkõ & -- & -- & -- & Sîkõ & Sîkũ & -- \\
\hline 411. & $* \sin a[n ? m V]$ & $\sin a ̃$ & şinã & sinán & -- & xinã & $\sin a \tilde{a}$ & $\sin a \tilde{a}$ \\
\hline 431. & *sopa[n?mV] & Şopã & Şopã & Şopán & -- & xopã & Şupã & Sopa \\
\hline 454. & *tiska[n?mV] & tişkã & tişskã & tişkán & -- & tixkã & tișkã & tişskã \\
\hline 464. & *tona[n?mV] & tonã & tonã & -- & -- & -- & tunã & tónã \\
\hline 470. & *wani[n?mV] & ßanĩ & wanĩ & wanín & -- & wanĩ & banĩ & -- \\
\hline 417. & *wara[n?mV] & ßarã & warã & warán & -- & wãrã & barã & фára \\
\hline 470. & *wani[n?mV] & ßanĩ & wanĩ & wanín & -- & wanĩ & banĩ & -- \\
\hline 471. & *wara[n?mV] & $\beta$ arã & warã & warán & -- & wãrã & barã & фárã \\
\hline 507. & *yom $[\mathrm{a}][\mathrm{n} 2 \mathrm{mV}]$ & omã & yomã & yomí & -- & yomã & yomi & yómi̊ \\
\hline
\end{tabular}

Nos casos em que, para Shell (1975), não havia o cognato em Chákobo, com base na observação das línguas Kashíbo, Shípibo-Kónibo e 
Kapanáwa - que tinham acento na sílaba mais à direita e na qual não havia nasalidade - reconstruiu-se uma sílaba a mais, representando-a por [CV].

Tabela 13 - Palavras trissilábicas reconstruídas por Shell (1975), cuja terceira sílaba terminava em consoante, mas sem especificaçáo da realizaçáo fonética da sílaba na protolíngua

\begin{tabular}{|c|c|c|c|c|c|c|c|c|c|c|c|}
\hline$\Sigma$ & 吾 & 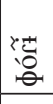 & . & $\begin{array}{l}0 \\
\frac{0}{20} \\
\frac{\pi}{2}\end{array}$ & 吾 & 卷 & 芯 & $\begin{array}{l}0 \\
\text { : } \\
0\end{array}$ & $\nsubseteq$ & : & 岂 \\
\hline$\overline{ \pm}$ & 苞 & $\overrightarrow{\vec{z}}$ & $\begin{array}{l}\vec{z} \\
\vec{\Xi}\end{array}$ & $\begin{array}{r}\vec{E} \\
\text { 吾 } \\
\end{array}$ & 洋 & $\overrightarrow{0}$ & $\stackrel{\widetilde{m}}{\vec{\omega}}$ & $\mathrm{i}$ & 志 & 洴 & 范 \\
\hline$\varangle$ & 荞 & i & $\begin{array}{l}8 \\
0 \\
0 \\
3\end{array}$ & i & 清 & 斈 & i & 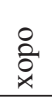 & $\nsubseteq$ & 清 & 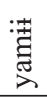 \\
\hline Eี & $i$ & 1 & 1 & 1 & $i$ & & $\stackrel{0}{0}$ & $\mathrm{i}$ & $i$ & : & $i$ \\
\hline 己े & 胥 & $\begin{array}{l}\text { 령 } \\
\text { ¿ }\end{array}$ & 码 & $\frac{0}{.0}$ & 暑 & 1 & 总 & 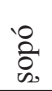 & 哭 & ! & 蒜 \\
\hline$\frac{v}{\sim}$ & 蛋 & $\begin{array}{l}\text { : } \\
\text { 。 }\end{array}$ & : & 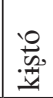 & 湿 & $\stackrel{\vec{c}}{\vec{\exists}}$ & 总 & $\begin{array}{l}\circ \\
\stackrel{\circ}{2} \\
0\end{array}$ & 急 & 洴 & 蔚 \\
\hline 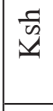 & 苨 & 莕 & $i$ & $\begin{array}{l}0 \\
0 \\
0 \\
\end{array}$ & 湴 & $\frac{\sqrt{0}}{3}$ & $\mathrm{i}$ & $\begin{array}{l}\circ \\
0 \\
0 \\
02\end{array}$ & : & $: \pm$ & $\vec{\Xi}$ \\
\hline 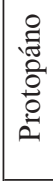 & 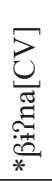 & 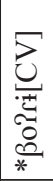 & 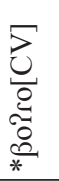 & $\begin{array}{l}3 \\
\frac{2}{0} \\
\frac{0}{0.0} \\
\frac{\pi}{*} \\
\end{array}$ & 总 & 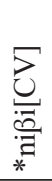 & 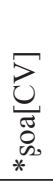 & $\begin{array}{l}\bar{z} \\
0 \\
0 \\
0 \\
0 \\
* \\
*\end{array}$ & 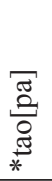 & $\underset{*}{\stackrel{D}{己}}$ & 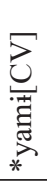 \\
\hline & i & $\dot{\alpha}$ & $\dot{\Xi}$ & $\dot{\infty}$ & ஸ் & $\frac{i}{\Delta}$ & $\ddot{\vartheta}$ & $\underset{\tilde{f}}{\tilde{f}}$ & チ่ & $\begin{array}{l}\dot{0} \\
\stackrel{7}{\sigma}\end{array}$ & சே \\
\hline
\end{tabular}


Notamos que, na tabela 13, o exemplo 423 não apresenta 3 sílabas em Chákobo e Shell (1975 [1965]) reconstruiu apenas com base nos reflexos de Shípibo-Kónibo e Kapanáwa, que possuem sílaba final tônica. Há outro caso em que a autora (1975 [1965]) reconstrói uma terceira sílaba, considerando apenas os reflexos em Káshíbo, Shípibo-Kónibo e Kapanáwa, pois a forma em Chákobo não apresenta 3 sílabas.

\section{Tabela 14 - Palavra trissilábica reconstruída para Protopáno sem reflexo trissilábico em Chákobo}

\begin{tabular}{|c|c|c|c|c|c|c|c|c|}
\hline & Protopáno & Ksh & SK & $\mathrm{Cp}$ & $\mathrm{Ch}$ & $\mathrm{A}$ & $\mathrm{Kn}$ & $\mathrm{M}$ \\
\hline 457. & *titõ $[\mathrm{CV}]$ & titõ & titõ & titón & tito & titõ & tituààku & tistõ \\
\hline
\end{tabular}

Girard (1971) traz importantes contribuiçôes para a discussão sobre formas nominais trissilábicas reconstruídas por Shell (1975 [1965]). Primeiramente, Girard (1971, p. 150) observa que Shell (1975 [1965]) "listou em seu conjunto de cognatos apenas as formas objetivas, exceto para o Chácobo onde aparentemente a forma ergativa é a forma de citação". Quanto às formas de marcação, o autor também observou que "a presença ou ausência dessa sílaba final pode bem sinalizar nas línguas contemporâneas a diferença entre ergativo e objetivo" (GIRARD, 1971, p. 150). ${ }^{3}$

A partir das observaçôes de Girard (1971), passamos a considerar que todas as línguas da família Páno apresentam formas longas e curtas para alguns nomes, o que discutiremos na próxima seção. Notamos que o uso dos termos "formas longas" e "formas curtas" pode ser mais neutro do que "formas ergativas e objetivas", uma vez que formas longas também podem ocorrer quando à raiz nominal se ajuntam outros sufixos casuais.

3 "[...] the presence or absence of this final syllable may well in the contemporary languages signal the difference between ergative and objective [...]." (GIRARD, 1971, p. 150) 


\title{
3 Formas longas e curtas em línguas páno
}

É importante ressaltar que foi Loos (1978, p. 135) $)^{4}$ o primeiro a estabelecer a diferença entre formas longas e curtas ${ }^{5}$ na família Páno, considerando que, nas línguas dessa família:

[...] um substantivo em função de sujeito tem uma forma fonológica mais longa que a forma do mesmo substantivo em funçáo de complemento direto, e a forma longa é, à primeira vista, a forma curta fusionada com o alomorfe de um sufixo marcador do sujeito (LOOS, 1978, p. 135) ${ }^{6}$.

Esta diferenciação é uma generalização descritiva que explica a alternância de formas como as que observamos nos dados da língua Kaxinawá7, abaixo, em que as palavras para "jacaré", "anta" e "onça” ocorrem ora com duas sílabas e ora com três sílabas.
1. kapi
huni
ka-î-shu-ki
jacaré.ABS
fugir/esconder
ir-?-PAS-?
'O jacaré fugiu'
2. kapitã huni kiyu-shu-ki jacaré.ERG homem morder-PAs-?
'O jacaré mordeu o homem'
3. awa huni ka-i-shu-ki anta.ABS fugir/esconder ir-?-PAS-?

'A anta fugiu'
4. awadin huni kiyu-shu-ki anta.ERG homem morder-PAs-? 'A anta mordeu o homem'

\begin{abstract}
${ }^{4}$ O leitor poderá perceber uma aparente incongruência nas datas das publicaçóes de Loos. No entanto, ao compararmos o texto que citamos como "Loos (1978)" com o texto que citaremos como "Loos (1973)", vemos que o texto publicado em 1978 está citado como publicado no texto de 1973. Esse fato nos levou a considerar o texto de 1978 como o primeiro cronologicamente, além de constituir indicaçấo de que o material a que tivemos acesso deve tratar-se de republicaçáo do texto original.

${ }^{5}$ No original, "formas largas" e "formas cortas" (cf. LOOS, 1978).

6 “[...] un substantivo en función de sujeto tiene una forma fonológica más larga que la forma del mismo substantivo en función de complemento directo, y la forma larga es, a primera vista, la forma corta fusionada con el alomorfo de un sufijo marcador del sujeto" (LOOS, 1978). O autor observa ainda que "en algunos de estos idiomas el sujeto intransitivo también lleva una marca, diferente del sujeto transitivo" (LOOS, 1978, p. 135).
\end{abstract}

${ }^{7}$ Agradeço a Joaquim Maná de Lima por, gentilmente, ceder-nos estes dados. 

5. huni
kamá
ui-shu-ki
homem.ERG
cachorro.ABS
ver-PAS-?
'O homem viu o cachorro'
6. kamã huni ka-i-shu-ki cachorro. ABS fugir/esconder ir-?-PAS-? 'O cachorro fugiu'
7. kamanì huni kiyu-shu-ki cachorro.ERG homem morder-PAS-?
'O cachorro mordeu o homem'

Loos (1978) estende o uso dos termos "formas longas" e "formas curtas" também às formas que não apresentam alternância no número de sílabas segundo a função sintática que desempenham. Nesse caso, a forma huni\& 'homem (ERG.)', acima, seria a forma longa de huni 'homem'. Para nós, seria mais adequado, no caso dos nomes das línguas Páno, reservar os termos formas longas e formas curtas apenas para as formas que apresentam alternância CVCV / CVCVCV.

A alternância entre formas longas e curtas, entretanto, ocorre também em alguns morfemas gramaticais de línguas Páno. Observe-se, por exemplo, o morfema comitativo -Bé / -Bétan, nos dados de Yawanawá, extraídos de Paula (2004, p. 204).
8. nani
Bitan kuni-n
atsa- $\emptyset$
pi-a
nome.próprio COM nome.próprio macaxeira-ABS comer-PAS
'Nani e Kuni comeram macaxeira'
9. audi- Ø-hin
auin-Baki-Bi
SuSu-pai-tiru
Aldir-ABS-FOC mulher-criança-COM brincar-DES-AF
'Aldir só quer brincar com sua filha'

Assim, como vimos nos nomes em Kaxinawá (exemplos de 1 a 8), o morfema comitativo em Yawanawá também apresenta duas formas, uma quando ocorre em sintagma nominal em função de A e outra quando ocorre em sintagma nominal em função de Sa (cf. PAULA, 2004).

$\mathrm{Na}$ etimologia 71, os reflexos deste morfema apresentam duas formas em praticamente todas as línguas, com exceção de Chákobo e Marúbo. Apenas em Korúbo, Matís e Mayorúna há reflexos de uma consoante final Ko Bét, Mt bidd e My bidd.

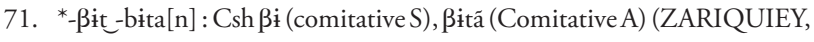


2011) : SC $-\beta \mathrm{i}--\beta \mathrm{itan}$ 'comitative' (VALENZUELA, 2003; LORIOT; LAURIAULT; DAY, 1993) : Cp - $\beta$ i $\sim-\beta$ ita 'con' (LOOS; LOOS, 1998) : Ch $\beta$ ita 'con' (ZINGG, 1998), bita 'en compañia de, con (sufijo nominal)'

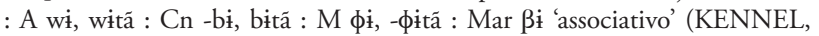
1978), - $\beta$ i 'associativo pronominal' (COSTA, 1992) : Kat -- : Shan fł_ fitan 'comitativo' ( $\mathrm{ft}$ 'comitativo com verbos intransitivos' fitan 'comitativo com verbos transitivos') : Yaw $\beta \dot{i} \_\beta$ itan 'comitativo' ( $\beta$ i 'intransitivas', Bitan 'transitivas') : Poy -- : Kax bí $~-$ bita 'comitativo' (VALENZUELA; OLIVEIRA, 2012) : Yam $\phi \dot{1}$, $\phi \dot{i t a}$ 'con' (EAKEN, 2008) : Chan $\phi \dot{i}, \phi i t a n$ 'con' : Shar :Ko ßit, ßita : Mt bid_bita_bitan 'comitativo' (bid 'S', bita 'O', bitan 'S') (FERREIRA, 2005): My bid_bita_bitan 'comitativo' (bid 'S', bita 'O', bitan 'S') (FLECK, 2003).

Concluímos, portanto, que também morfemas gramaticais apresentam formas longas e curtas e que essa alternância se relaciona diretamente com a função sintática dos sintagmas, paralelamente ao que ocorre nos nomes.

Dessa forma, redefinimos formas longas como a maior forma de um nome ou de um morfema gramatical que apresenta duas formas alternantes condicionadas pela sua função sintática; e a forma curta, por sua vez, seria a menor forma de um nome ou de um morfema gramatical que apresenta duas formas alternantes condicionadas pela sua função sintática.

Quanto aos verbos, Loos afirma que:

[...] o mesmo processo de encurtamento que reduziu os substantivos de forma CVCVCV do Protopáno se aplicou a raízes verbais, mas a existência de sufixos vocais-iniciais pode ter ajudado a conservar as originais terceiras consoantes de raízes CVCVC(V) (LOOS, 1978, p. 166) ${ }^{8}$.

É bastante provável que Loos (1978) utilize a representação de trissilábicas em Protopáno, com base na reconstrução de Shell (1975 [1965]), que postulou formas trissilábicas em todos os casos em que os nomes em Chákobo apresentavam a forma CVCVCV, o que discutiremos mais adiante. $\mathrm{O}$ mais importante, entretanto, é que seria possível que o processo de alternância entre formas longas e curtas ocorresse tanto em nomes quanto em verbos nas línguas da família Páno. Assim sendo, em sentido mais amplo, poderíamos dizer que verbos também possuem formas longas e curtas. No entanto, o condicionamento de ocorrência das formas longas e curtas de um verbo seria diferente do condicionamento de formas longas e curtas dos nomes e dos morfemas gramaticais. Voltaremos a esse

8 “[...] el mismo proceso de acortamiento que redujo los substantivos de forma CVCVCV del proto-pano se aplicó a raíces verbales, pero la existencia de sufijos vocales-iniciales puede haber ayudado a conservar las originales terceras consonantes de raíces CVCVC(V)". 
tema na próxima seção deste capítulo. Por ora, acreditamos que as definiçôes até então mobilizadas são suficientes para o entendimento da discussão desenvolvida nas seções subsequentes.

\subsection{Reconstruçáo de consoantes em margem direita de sílaba em Protopáno}

Ao discutir o sistema de marcação de caso nos nomes, Córdoba, Valenzuela e Villar (2011, p. 13) observam que há em Chákobo uma alternância entre formas longas e formas curtas, parcialmente condicionada pela função sintática do nome e também pela sua posição na oração. As formas curtas ocorrem apenas em funçăo de objeto $(\mathrm{O})$ ou de sujeito de intransitiva $(S)$ e em posição pré-verbal, enquanto as formas longas ocorrem em posição de $\mathrm{O}$, de $\mathrm{A}$ e de $\mathrm{S}$, em posição pré e pós-verbal. Córdoba, Valenzuela e Villar (2011, p. 21) observam também que "sólo las [formas] trissilábicas ocurren como formas de citación".?

Os dados seguintes, extraídos de Córdoba, Valenzuela e Villar, (2011, p. 21), ilustram o comportamento dessas formas em Chácobo. A análise é a mesma apresentada no artigo original.

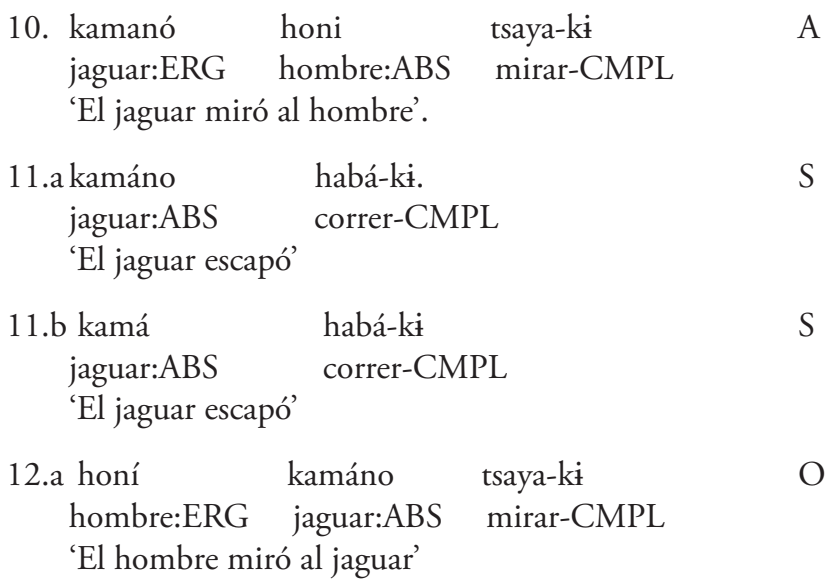

\footnotetext{
${ }^{9}$ Loos (1978) também havia observado a presença de formas longas e curtas em Chákobo.
} 

12.b honí kamá tsaya-ki hombre:ERG jaguar:ABS mirar-CMPL 'El hombre miró al jaguar'

$\mathrm{O}$
13. habá-ki kamáno correr-CMPL Jaguar.ABS 'El hombre miró al jaguar'

A partir da observação de formas longas e curtas, em línguas da família Páno e, principalmente, em Chákobo, postulamos que a protolíngua também teria formas longas e curtas e que as formas trissilábicas reconstruídas por Shell (1975 [1965]) são, na verdade, formas flexionadas para caso.

No caso da língua Chákobo, diferentemente das outras línguas, haveria uma mudança em curso, em que as formas longas deixaram de ser interpretadas como formas flexionadas e passaram a ocorrer também na função de $\mathrm{O}$ e de $\mathrm{S}$. No entanto, a língua Chákobo ainda mantém resquícios do sistema original, que náo permitem que formas curtas ocorram na função de A (também LOOS, 1978).

Dados das línguas Korúbo, Matís e Mayorúna nos dão as evidências adicionais de que as formas curtas das palavras apresentadas na tabela 11 teriam uma consoante em final de palavra na protolíngua. Na tabela a seguir, apresentamos as nossas reconstruçóes de palavras do Protopáno ilustradas também com os cognatos nas línguas Korúbo, Matís e Mayorúna. Ressaltamos que, na tabela seguinte, as formas da língua Chákobo são todas formas curtas. 
Tabela 15 - Formas curtas com consoante final

\begin{tabular}{|c|c|c|c|c|c|c|c|c|c|c|c|c|c|c|c|c|c|c|}
\hline$\vec{\Sigma}$ & 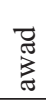 & $\frac{3}{2}$ & รీ & & . & $\begin{array}{c}n \\
0 \\
0 \\
0\end{array}$ & 1 & 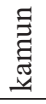 & 1 & 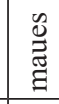 & 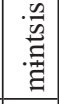 & $i$ & I & 苟 & $\begin{array}{l}\text { 胥 } \\
\text { 吾 }\end{array}$ & $\begin{array}{l}\text { 吾 } \\
\stackrel{\Xi}{\Xi}\end{array}$ & 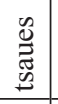 & \\
\hline$\sum^{E}$ & $\begin{array}{l}\text { 芯 } \\
\text { 尝 }\end{array}$ & 龵 & & & $\stackrel{\varrho}{\Xi}$ & $i$ & 楛 & 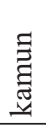 & 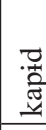 & 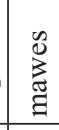 & 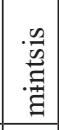 & \begin{tabular}{|l|} 
吾 \\
莦 \\
\end{tabular} & 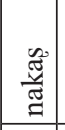 & 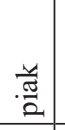 & 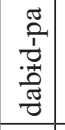 & 1 & 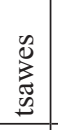 & 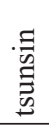 \\
\hline$\stackrel{\otimes}{ }$ & 莺 & 局 & 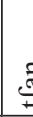 & & 1 & $i$ & 楛 & $\begin{array}{l}\text { 音 } \\
\text { 昰 }\end{array}$ & 1 & i & 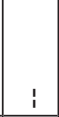 & 1 & 1 & 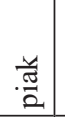 & 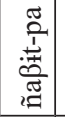 & i & $\begin{array}{l}0 \\
0 \\
\sum_{0}^{0} \\
0 \\
0\end{array}$ & 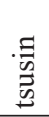 \\
\hline$\Sigma$ & : & & 28 & & $i$ & $\begin{array}{l}\overrightarrow{0} \\
\stackrel{0}{0}\end{array}$ & 港 & 点 & 试 & : & 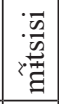 & $\frac{\vec{*}}{\vec{a}}$ & 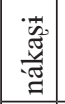 & $\frac{\pi}{2}$ & 蘦 & 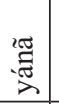 & 菷 & $\frac{5}{2}$ \\
\hline$\Xi$ & 䓠 & & 29 & & 1 & 言 & 词 & 营 & 善 & : & 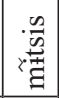 & 泀 & 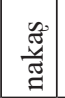 & $i$ & $\begin{array}{l}\text { 莞 } \\
\vec{\sigma}\end{array}$ & 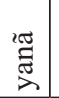 & $\stackrel{\vec{\nexists}}{\triangle}$ & $\stackrel{5}{2}$ \\
\hline$\ll$ & 唸 & i匹 & 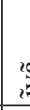 & & $\stackrel{n}{s}$ & 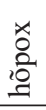 & 苦 & 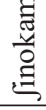 & $\begin{array}{l}: \text { 泀 } \\
\end{array}$ & 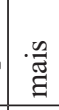 & 足 & 萻 & 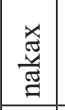 & : & 汶 & 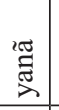 & $\stackrel{\overparen{D}}{\triangle}$ & $\frac{5}{2}$ \\
\hline छ & 莺 & 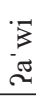 & הִ & & $\stackrel{n}{\Delta}$ & 峁 & 沽 & 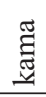 & 芼 & 1 & 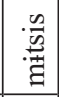 & 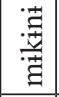 & t & $\stackrel{\pi}{\overparen{2}}$ & 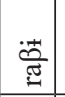 & $\underset{\overbrace \widetilde { \pi }}{\overparen{\sim}}$ & $i$ & $\stackrel{-}{\circ}$ \\
\hline قे & $\begin{array}{l}\pi \\
\pi \\
\pi \\
\pi\end{array}$ & $\begin{array}{l}.5 \\
-\sigma\end{array}$ & 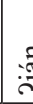 & & i & 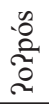 & I & i & $\begin{array}{l}\text { : 荡 } \\
\text {. }\end{array}$ & i & 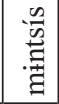 & 吾 & 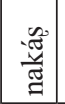 & $\begin{array}{l}\frac{\pi}{\mathbb{Z}} \\
\stackrel{\widetilde{a}}{2}\end{array}$ & 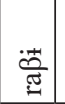 & 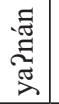 & 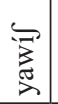 & $\stackrel{\Xi}{2}$ \\
\hline$\frac{1}{n}$ & $\begin{array}{l}\pi \\
\pi \\
\pi \\
\pi\end{array}$ & $\begin{array}{l}3 \\
-3 \\
-\sigma\end{array}$ & ?ְ. & & $\mathrm{i}$ & $\begin{array}{l}0 \\
0 \\
0 \\
0\end{array}$ & 范 & 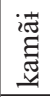 & 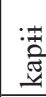 & 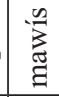 & 证 & 蒙 & 跑 & $: \frac{\pi}{2}$ & 节 & 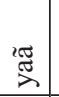 & 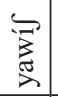 & $\stackrel{5}{\circ}$ \\
\hline ज्ञ & $\therefore$ & i & & & : & 1 & 嵒 & 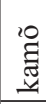 & $\overrightarrow{\mathrm{a}}$ & 呪 & $i$ & 蓄 & 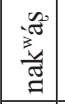 & $\begin{array}{l}\frac{\pi}{\pi} \\
\frac{\pi}{2} \\
\end{array}$ & 节 & '표 & $\bar{\pi}$ & 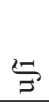 \\
\hline & 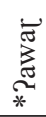 & 罣 & & & . & 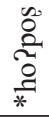 & $\frac{\Xi}{*}$ & 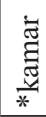 & 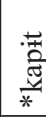 & $\underbrace{n}_{*}$ & 竧 & 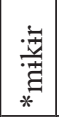 & 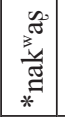 & 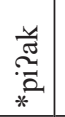 & 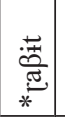 & $\underset{*}{\stackrel{\Xi}{*}}$ & $\sum_{*}^{\infty}$ & $\begin{array}{l}\text {.7 } \\
\text { ह } \\
* \\
*\end{array}$ \\
\hline & & & & & & & & & & & & & an & & ci & gु & g. & $\bar{n}$ \\
\hline
\end{tabular}


Observando a tabela 15, podemos notar que apenas Korúbo, Matís e Mayorúna mantiveram as consoantes oclusivas (incluindo $n)^{10} \mathrm{em}$ final de palavra nas formas curtas, enquanto as demais línguas perderam as consoantes oclusivas nessa posição. Todas as línguas mantiveram as consoantes fricativas em final de palavra.

As formas longas e curtas em Chákobo, Amawáka, Iskonáwa, Sharanáwa, Kaxinawá, Shípibo, Kashíbo, Kapanáwa e Mayorúna são analisadas por Loos (1978), a partir de uma regra de apagamento de consoantes oclusivas (incluindo $m$ e $n$ ), a qual ele acreditava existir na protolíngua, e estabelece regras sincrônicas para as seguintes línguas:

Chákobo: "[...] se suprimem consoantes oclusivas que precedem a outra consoante ou a margem da palavra. Incluímos consoantes nasais no grupo de oclusivas. As consoantes fricativas náo serão afetadas por esta regra" (LOOS, 1978, p. 141) ${ }^{11}$.

Amawáka: “[...] suprimir consoantes oclusivas (inclusive $m$ e $n$ ), quando são finais de sílabas" (LOOS, 1978, p. 145). ${ }^{12}$

Sharanáwa: "[...] suprimir consoantes não contínuas finais de sílaba ou antes de uma juntura de morfemas" (LOOS, 1978 p. 149)..$^{13}$

Shípibo: "[...] suprimir consoantes oclusivas (inclusive nasais) ao final de sílaba” (LOOS, 1978, p. 151) ${ }^{14}$.

O autor analisa, de forma semelhante, as línguas Iskonáwa, Kaxinawá, Kashíbo e Mayorúna. Entretanto, notamos que, nos dados apresentados por Loos (1978) para a língua Mayorúna, não há nenhuma palavra que apresente a alternância entre formas longas e curtas, o que o leva a concluir que, em Mayorúna, "as formas longas se perderam completamente" (LOOS, 1978.). Diferentemente de Loos (1978; 1973), vemos que não só a língua Mayorúna, mas as línguas Matís e Korúbo (que são geneticamente mais próximas da primeira) apresentam uma alternância entre formas longas e

\footnotetext{
${ }^{10}$ Provavelmente o fonema ${ }^{*} \mathrm{r}$ da protolíngua era mais oclusivo quando em final de palavra, o que explicaria o seu apagamento nesse contexto.

11 “[...] se suprimen consonantes oclusivas que preceden a otra consonante o el margen de la palabra. Incluímos consonantes nasales en el grupo de oclusivas. Las consonantes fricativas no serán afectadas por esta regla." (LOOS, 1978op. cit., p. 141 p. 141)

12 "[...] suprimir consonantes oclusivas (inclusive $\mathrm{m}$ y n) cuando son finales de sílabas." (op. cit., p. 145)

13 “[...] suprimir consoantes no continuas final de sílaba o ante una juntura de morfemas." (op. cit., p. 149)

14 "[...] suprimir consonantes oclusivas (inclusive nasales) al final de sílaba." (LOOS, 1978op. cit., p. 141, p. 151)
} 
curtas, assim como, também, são as únicas a apresentar uma consoante em final de palavra nas formas curtas. Essas observaçóes nos levaram a considerar não só a reconstrução dessa alternância para a protolíngua, como também uma consoante final de palavra em suas formas curtas.

Abaixo, apresentamos alguns exemplos de alternância entre formas longas e curtas na língua Matís, em que a forma curta apresenta consoante final. Apresentamos, em seguida, dados de Ferreira (2005), com algumas adaptações na segmentação e na análise para evidenciar as diferenças entre as formas longas e curtas. Escolhemos exemplos da língua Matís, por esta conservar cognatos que aparecem nos dados compartilhados por outras línguas e que ilustram melhor a discussão.

$\begin{array}{lll}\text { 14. awadin } & \text { tumi } & \text { nidikabos } \\ \text { awad-in } & \text { tumi } & \text { nidika-bo-S } \\ \text { anta-erg } & \text { nome.próprio } & \text { correr.atrás.de-pass.n.rec-? } \\ \text { 'A anta correu atrás do Tumi' } & \end{array}$

15. kodokakidin awad kodokaek kodoka-kid-in awad kodoka-e-k cozinhar-nmlz-erg anta cozinhar-n.pass-?

'O cozinheiro cozinha a anta'

16. kamunin unkin akkid kamun-in unkin ak-kid onça-erg caititu matar-nmlz 'A onça ì a que mata caititu'

17. nimuduk kamun abi nimuduk kamun abi floresta onça existencial 'Na floresta tem onça'

18. kamun matses-in pe-kimo-esma onça Matis-erg comer-inten-neg. 'Os Matís nunca (mesmo) comem onça'

Em todos os exemplos apresentados, a diferença entre forma longa e forma curta se deve a uma forma em função de A (longa) e uma forma em função de $\mathrm{O}$ ou de $\mathrm{S}$ (curta). No entanto, há também exemplos, nas várias línguas, em que a diferença se deve a uma forma flexionada para outros 
casos, como o instrumental ou o genitivo. No exemplo seguinte, a palavra awad 'anta', do Matís, aparece em sua forma longa, flexionada para caso genitivo:

$\begin{array}{llll}\text { 19. inbi } & \text { awadin } & \text { tai } & \text { isak } \\ \text { inbi } & \text { awad-in } & \text { tai } & \text { is-a-k } \\ \text { 1 sg.erg } & \text { anta-gen } & \text { pi } & \text { ver-pass.rec-decl } \\ \text { 'eu vi a pegada da anta' (lit. o pi da anta) }\end{array}$

A proposta que apresentamos é a de que a protolíngua possuía consoantes em margem direita de sílaba nas formas curtas e que as línguas Kashíbo, Shípibo-Kónibo, Kapanáwa, Chákobo, Amawáka, Kaxinawá, Marináwa, Katukína, Shanenáwa, Yawanawá, Poyanáwa, Yamináwa, Chanináwa e Sharanáwa, antes de silêncio, perderam todas elas, exceto as consoantes fricativas. As únicas línguas que preservaram consoantes oclusivas em margem direita de sílaba (incluindo nasais) antes de silêncio foram Matís, Mayorúna e Korúbo. O caso da língua Kaxararí será discutido em seção específica.

Propomos, então, que as consoantes finais de palavra em Kashíbo, Shípibo-Kónibo, Kapanáwa, Chákobo, Amawáka, Kaxinawá, Marináwa, Katukína, Shanenáwa, Yawanawá, Poyanáwa, Yamináwa, Chanináwa e Sharanáwa ainda fazem parte da estrutura sonora de certas formas, mas estas consoantes só se realizam fonologicamente quando um sufixo iniciado por vogal é adicionado a elas.

Consoantes oclusivas em margem direita de sílaba também são reconstruíveis para a protolíngua em morfemas gramaticais, como mostram os exemplos 52, 71, 418 e 419.

52. *hatit: Csh atian 'then' (ZARIQUIEY, 2011) : SC ha'ti 'tanto' (LORIOT; LAURIAULT; DAY, 1993), hatíßĩ : Cp hati?ß̃̃ 'todos' : Ch ha'ti 'toditos, todo igual' (ZINGG, 1998), hatíta 'todo : A -- : Cn hatibi 'todos esos, todos; cada uno' : M nãitiøĩi : Mar aatí 'essa quantia' (KENNEL, 1978), ati [' āti*] '3Pl (esses) (COSTA, 1992) : Kat -- : Shan -ti 'quantificador' : Yaw aui-ti 'quanto' : Poy -- : Kax -- : Yam -tii 'todos, varios del mismo tipo, esta cantidad' (FAUST; LOOS, 2002; EAKEN, 2008) : Chan natiфi 'todos', datian 'ahora' : Shar atişon 'de este tamaño (objeto entero)' : Ko atet 'esse tanto' : Mt ted 'tantos quantos' (FERREIRA, 2005) : My ted 'as many as' (FLECK, 2003), tedi 'all of' : (Csh kamaßi, ver Mar abajo). 
71. $\quad *-\beta$ it $\infty-$ bita[n] : Csh $\beta$ i (comitative S), $\beta$ itã (comitative A) (ZARIQUIEY, 2011) : SC - $\beta \dot{\mathbf{i}} \sim-\beta i \tan$ 'comitative' (VALENZUELA, 2003; LORIOT; LAURIAULT; DAY, 1993) : Cp - $\beta$ i - $\beta$ ita 'con' (LOOS; LOOS, 1998) : Ch ßita 'con' (ZINGG, 1998), bita 'en compañia de, con (sufijo nominal)' : A wii 'con' (HYDE, 1980), wi, witã : Cn -bi, bitã : M Fi, -Fitã : Mar ßi 'associativo' (KENNEL, 1978), - $\beta \dot{i}$ 'associativo pronominal' (COSTA, 1992) : Kat -- : Shan fi / fitan 'comitativo' (fi 'comitativo com verbos intransitivos' fitan 'comitativo com verbos transitivos') : Yaw $\beta \dot{i} / \beta$ itan 'comitativo' ( $\beta \dot{i}$ 'intransitivas', $\beta$ itan 'transitivas') : Poy -- : Kax bi $\sim$ bita 'comitativo' (VALENZUELA; OLIVEIRA, 2012) : Yam Fi, Fita 'con' (EAKEN, 2008) : Chan Fi, Fitan 'con' : Shar -- : Ko ßit, ßita : Mt bid / bita / bitan 'comitativo' (bid 'S', bita 'O', bitan 'S') (FERREIRA, 2005) : My bid / bita / bitan 'comitativo' (bid 'S', bita 'O', bitan 'S') (FLECK, 2003).

418. *-so[n]: Csh sun 'benefactive applicative' (ZARIQUIEY, 2011), -ş̃̃ : SC - șõ : Cp - șon : Ch -- : A xõ : Cn -șuàà : M -șõ 'benefactivo' : Mar șo sõ 'benefício' (KENNEL, 1978) : Kat -șu\& (-shõ) 'benefício' (MENDES, 1998) : Shan -șun, șuna 'benefactivo' : Yaw -Sun 'benefactivo' : Poy -- : Kax -- : Yam șon 'benefactivo' (FAUST; LOOS, 2002) : Chan -- : Shar -son- 'benefactivo' : Ko -Sun 'benefactivo' : Mt - sun 'Morfema verbal benefactivo' (FERREIRA, 2005) : (Ch -şi 'benefactivo futuro').

419. *-son : Csh -şun (ZARIQUIEY, 2011), -șõ : SC -ş̃̃ : Cp -şon

: Ch şo : A - xoàà : Cn - şuàà : M -- : Mar şo şõ (KENNEL, 1978)

: Kat şõ'subordinação transitiva, aspecto completo' (MENDES, 1998)

: Shan -șun 'marcador de switch reference transitivo' : Yaw -- : Poy -- : Kax -Su Sun (VALENZUELA; OLIVEIRA, 2012) : Yam -șõ : Chan -- : Shar -- : Ko șun : Mt - șun 'morfema de concordância de transitividade' (FERREIRA, 2005) : My -șun : 'sufijo de concordância transitiva'.

A ocorrência de consoantes em margem direita de sílaba, tanto em palavras quanto em morfemas gramaticais, sugere que a perda de consoantes oclusivas em margem direita de sílaba - em Kashíbo, ShípiboKónibo, Kapanáwa, Chákobo, Amawáka, Kaxinawá, Marináwa, Katukína, Shanenáwa, Yawanawá, Poyanáwa, Yamináwa, Chanináwa e Sharanáwa - deve ser resultado de uma reestruturação dos padróes silábicos, nessas línguas. Nos exemplos abaixo, extraídos de Ferreira (2005), observamos que Matís, uma das línguas que manteve consoantes oclusivas em final de palavra, também apresenta essas consoantes em meio de palavra e em margem direita de sílaba. 

a) /andadawid/
b) /iksamadap/
c) / $\mathrm{iksak} /$
d) edta
andadawid
iksama-dap
ikșak
ed-ta
'vazio' 'ruim-?'
'boca'
'Entre!'

Em Protopáno, as consoantes oclusivas teriam ocorrido em margem direita de sílabas em verbos, como ilustrado pelas etimologias 1 e 110, reproduzidas aqui:

1. *Rak-: Csh Pa- : SC Pa- : Cp Pa- : Ch Pa- : A Pa- (SPARINGCHAVEZ, 2012) : Cn a- : M a- 'hacer' : Mar a : Kat -- : Shan -- : Yaw -- : Poy -- : Kax a- : Yam ak- Chan a- : Shar a- 'hacer, matar' : Ko ak-, Mt ak- : My ak-.

110. *tsa?o[t]- : Csh tsoot 'live' (ZARIQUIEY, 2011), tsóti 'sentarse', tsónti 'sentar' (SHELL, 1987), tsOOi : SC tsaóti, tsaóta 'ponerse de coclillas' (LORITO; LAURIAULT; DAY, 1993) : Cp tsa?ó- 'sentado, asentado' tsa?o-'ti 'sentarse' : Ch tsa?o- 'sentarse' : A tsa?oo-Pi 'sentarse' (HYDE, 1980), tsa?o- II : Cn tsau- 'sentarse', tsaua 'sentado' : M tsáo- : Mar tsao- 'sentar' (CESARINO, 2008) : Kat -- : Shan tsaw 'sentar' : Yaw tsau 'sentar' : Poy -- : Kax -- : Yam tsao (FAUST; LOOS, 2002) : Chan tsao- : Shar tsaoi, tsaoa 'sentarse' : Ko tsat : Mt tsad : My tsad 'sentarse'.

A consoante $t$ final de verbos é analisada por Valenzuela (2013) como um morfema que intransitiviza verbos em Shípibo, Kashíbo e Matsés. Entretanto, é muito provável que a consoante $t$ final fizesse parte da raiz e se apagasse ao receber morfemas iniciados por consoantes, manifestandose fonologicamente com a adição de sufixos iniciados por vogais. A autora (VALENZUELA, 2013.) apresenta os seguintes exemplos que demonstram a possível oposição entre os morfemas - $t$ 'intransitivizador' e -n 'transitivizador em Shípibo.

$\begin{array}{lll}\text { raka-t- 'echarse' } & v s . & \text { raka-n- 'echar algo/a alguien' } \\ \text { wini-t- 'pararse' } & v s . & \text { wini-n- 'parar algo/a alguien' } \\ \begin{array}{l}\text { tsao-t- 'ponerse de cuclillas' } \\ \text { algo/a alguien' }\end{array} & v s . & \text { tsao-n- 'poner de cuclillas } \\ \text { yaka-t- 'sentarse' } & v s . & \text { yasa-n- 'sentar algo/a algui }\end{array}$

Note-se que, em Shípibo, o verbo paké 'cair', apresenta um $t$ quando seguido de morfema constituído por vogal, porém, quando seguido do morfema - $k$ é, o $t$ não ocorre. 


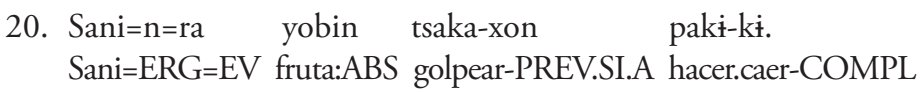
'Sani le dio a la fruta (con flecha, piedra o palo) y la hizo caer (del árbol)'.

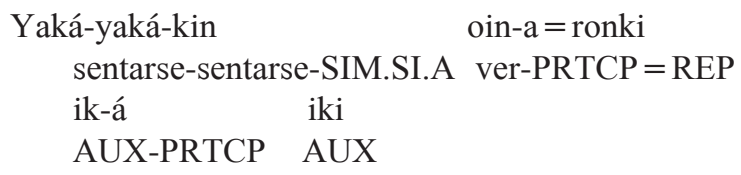

Embora reconstruamos também consoantes em margem direita no final de alguns verbos, vemos a possibilidade de que, nos exemplos precedentes, o - $t$ seja parte do tema, mas náo necessariamente da raiz.

\subsection{Natureza fonética das consoantes em margem direita de sílaba}

Ao compararmos as consoantes finais das formas curtas das línguas Korúbo, Matís e Mayorúna, verificamos que elas se diferenciam das consoantes encontradas nas formas longas correspondentes de outras línguas. Isso nos levou a considerar que essas três línguas inovaram.

\subsubsection{Reflexos de ${ }^{*} t \mathrm{e}^{*} r$ em final de palavra}

Nas etimologias em 8 e 171, verificamos que os reflexos da consoante final * ${ }^{*}$ e da consoante final ${ }^{*} t$ são $d$ em Matís. No entanto, o reflexo de *r na forma longa em Shípibo-Kónibo e Chákobo é $r$, e na forma longa em Kaxararí é $t S$. Já em 171, o reflexo de ${ }^{*} t$ na forma longa em Chákobo, Yamináwa e Kaxararí é $t$, e, embora seja também $t$ em Korúbo, é $d$ em Matís e Mayorúna. 
8. *?awar: Csh ?O'O : SC Ra'wa 'tapir', ?awarã piti 'comida de tapir (nombre de planta)' : Cp ?a'wa; Ch Páwara 'tapir’ A ?áá (HYDE, 1980), áá I : Cn awa : M áwa : Mar awa : Kat awa [aw'a] (BARROS, 1987) : Shan awa : Yaw aua : Poy awa : Kax [awa'tSa] : Yam aфa (FAUST; LOOS, 2002) : Chan -- : Shar awa, awa-pan : Ko awat : Mt awad : My awad.

171. *kapit : Csh kapi [ka'pi] (ZARIQUIEY, 2011), kapi : SC kapii : Cp kapii : Ch 'kapiti, 'kapi 'caimán, lagarto' (ZINGG, 1998), kápiti A kápii (HYDE, 1980), kapii I : Cn kapi : M kápi\&\& : Mar ka'pi (ANONBY; HOLBROOK, 2010) : Kat ka'pi : Shan kapi : Yaw kapi : Poy kapi : Kax kapi'ti : Yam kapi, kapita 'lagarto' (EAKEN, 2008) : Chan kapi 'lagarto' : Shar kapi, kapitan 'lagarto, caimán' : Ko -- : Mt kapid 'jacari’ : My -- : 'lagarto'.

Como os reflexos regulares de ${ }^{*} r$ é $r$ em Shípibo-Kónibo e Chákobo e é $t S$ em Kaxararí, consideramos que a protoforma seria * em 171, que os reflexos em Korúbo seriam $t$, e os reflexos em Matís e Mayorúna seriam $d$, nessa posição. Da mesma forma, na etimologia 171 , o reflexo de proto ${ }^{*} t$ em final de palavra é também $t$ em Korúbo e $d$ em Matís e Mayorúna. Mas, nesse caso, reconstruímos um Protopáno ${ }^{*} t$, com base nas formas longas do Chákobo, do Yamináwa e do Kaxararí, as quais apresentam $t$, nessa posição.

Da discussão acima, conclui-se que, em final de palavra, em margem direita de sílaba, Korúbo fundiu os reflexos de ${ }^{*} t \mathrm{e}{ }^{*} t$, ambos resultando em $t$. Já em Matís e Mayorúna, os reflexos de ${ }^{*} t \mathrm{e}^{*} r$ também se fundiram, mas mudaram posteriormente para $d$.

Reconstruímos, dessa forma, uma consoante coronal oral, representada por $C_{c}$, sempre que há um reflexo $t$ em Korúbo ou $d$ em Matís e Mayorúna, em final de palavra, e sempre que há acento final em Kashíbo, ShípiboKónibo e Kapanáwa, embora não tenhamos identificado reflexos nas formas longas das demais línguas, como ilustra a etimologia 99, a seguir:

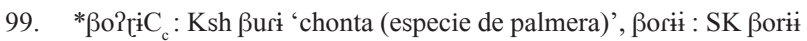
: Kp ßo?rï : Ch -- : A -- : Kn budi : M Fóri : Mar -- : Kat -- : Shan Furi 'palmeira' : Yaw -- : Poy -- : Kax -- : Yam -- : Chan -- : Shar Fori, Forin 'chonta (espicie de palmera y su cogollo comestible)' : Ko ßoñit 'palmeira que utilizam para cobrir a casa (prov. Jarina)' : Mt -- : My budid [bu'cid] 'palmera grande'.

Reconstruímos, também, uma consoante $\mathrm{C}$, quando não possuíamos dados que evidenciassem os reflexos em Korúbo, Matís e Mayorúna, mas havia acento na última sílaba em Kashíbo, Shípibo-Kónibo e Kapanáwa. 


\subsubsection{Reflexos de ${ }^{*} m,{ }^{*} n$ e ${ }^{*} r$ em final de palavra}

Como vimos, somente Kaxararí não fundiu os reflexos de Protopáno ${ }^{*} r$ e ${ }^{*} n$, mantendo $l$ como reflexo de ${ }^{*} r$ e $n$ ou $V \&$ como reflexo de ${ }^{*} n$. A reconstruçáo de um proto ${ }^{*} r$ ou de um proto ${ }^{*} n$, em margem direita de sílaba final de palavra, foi feita com base nos reflexos das formas longas do Kaxararí, como se observa nas etimologias a seguir:

165. *kamar: Csh kamõ 'perro (siendo remplazado por el pristamo $\mathrm{SC}$, /otSíti, que es a su vez probablemente um pristamo del campa otfiti)' : SC kamãi 'demonio, criatura parecida al tigre' (/otfíti 'perro') : Cp kaman /ino 'sachaperro (esp. de perro silvestre)' (Cp /ot fíti 'perro') : Ch ka'mano, kama 'el tigre, el jaguar' (ZINGG, 1998), kamáno 'felino' : A káman, kámanin 'esp. de roedor como majás', Sinokamã 'perro salvaje' : Cn kamã 'perro' : M kámã 'perro' : Mar ka'mã (ANONBY; HOLBROOK, 2010) : Kat ka'man : Shan kaman 'cachorro' : Yaw kaman 'cachorro' : Poy kãma 'raposa' : Kax kamalu [kama'lu] [kCE\&ma'lu] (VALENZUELA; OLIVEIRA, 2012) : Yam -- : Chan -- : Shar kaman, kamanon 'perro' : Ko kamun 'onça' : Mt kamun 'onça' : My kamun [ka.'mun] 'jaguar'.

Em 165, reconstruímos ${ }^{*} r$ considerando o reflexo $l$ na forma longa da língua Kaxararí. Já as línguas Korúbo, Matís e Mayorúna possuem $n$ como reflexo de ${ }^{*} r$.

$\mathrm{Na}$ etimologia 9, reconstruímos uma consoante alveolar ${ }^{*} n$, com base no reflexo $n$ da forma longa em Kaxararí e Chákobo.

94. *ßinon : Csh ßinõi : SC ßinó : Cp ßinon 'aguaje, palma real' : Ch 'ßinona, ßino 'la palma real' : A winõ I : Cn -- : Mar ßinõ 'buriti' (CESARINO, 2008) : Kat $\beta$ inu [vi\&o/] ( $\beta$ ARROS, 1987) : Shan -- : Yaw ßinun- : Poy -- : Kax binu'ni [binu'ni] [bi'nui] (LANES, 2005) : Yam -- : Chan -- : Shar -- : Ko -- : Mt -- : My -- : 'clase de palmera'

Para a reconstrução de ${ }^{*} m$ em final de palavra, consideramos as formas longas de outras línguas, principalmente do Chákobo. Em 86, por exemplo, Chákobo apresenta $m$ em sua forma longa, mas o som correspondente em Korúbo e Matís é $n$.

86. * $\beta$ itim : Ksh $\beta$ iti\& 'cocinar carne en agua' : SK $\beta$ iti\& 'guiso de pescado' : Kp ßitin 'sopa' (LOOS; LOOS, 1998) : Ch ßitiimi 'alimento' : A witi\& II 'bebida hecha de maíz dulce' : Kn biti\& 'sopa que contiene 
carne' : M Fiiti\& 'sopa que contiene carne' : Mar -- : (Kat yuntu 'sopa de peixe') : Shan -- : Yaw -- : Poy -- : Kax -- : Yam -- : Chan -- : Shar Fitin 'sopa' : Ko ßitin 'sopa, caldo, mingau' : Mt bitin 'sopa' : My --.

A partir da análise desses casos, foi possível postular a ocorrência em Korúbo, Matís e Mayorúna de uma fusão em $n$ dos reflexos de proto ${ }^{*} m$, ${ }^{*} n$ e ${ }^{*} r$, em margem direita de sílaba final de palavra.

Reconstruímos, assim, uma consoante nasal *[n], quando há ausência de dados do Kaxararí ou de formas longas nas demais línguas; há, todavia, apenas reflexos nasais em margem direita de sílaba final nas demais línguas.

3. /ami[n]: Ksh /a'mi̊\&: SK /a'mi̊\&: $\mathrm{Cp} / \mathrm{a}$ 'min : Ch amino, ami ‘la capyguara' : A /amin (HYDE, 1980) : Kn ami̊ : M 'ami̊\& : '/amino 'capibara' : Mar ami (MNTB-LOOS, s.d.) : Kat amin : Shan aman : Yaw aman : Poy -- : Kax -- : Yam -- : Chan -- : Shar amin : Ko -- : Mt -- : My --.

344. *p[õ]si[n] : Ksh posiàà : SK põsiàà : Cp -- : Ch -- : A posiàà I : Kn : M pósiàà : Mar -- : Kat punsi : Shan pusan [pu'sã\&] : Yaw -- : Poy -- : Kax -- : Yam -- : Chan -- : Shar -- : Ko pusin 'esp. de preguiça' : Mt pusin 'preguiça real' : My posin 'peresozo de dos dedos' : 'clase de perezoso'.

\section{As formas longas e curtas em Kaxararí}

A língua Kaxararí parece não possuir mais um sistema de oposição funcional entre formas longas e curtas. Entretanto, tal como ocorre com o Chákobo, as formas analisadas em Kaxararí apresentam sempre reflexos de formas longas, como é possível ver nas etimologias, a seguir, extraídas de Valenzuela e Oliveira (2012):

\begin{tabular}{ll}
\multicolumn{2}{l}{ Formas longas em Kaxararí } \\
Kaxarari & Português \\
awatsa & 'anta' \\
kapiti & 'jacarì' \\
yawiSi & 'tatu' \\
mikili & 'mão'
\end{tabular}


Nos poucos dados que conseguimos até o presente, as formas longas da língua Kaxararí também ocorrem em funçáo de $\mathrm{O}$, como vemos, em seguida, na palavra "anta", mas nossa hipótese é a de que a vogal deve ser reflexo de algum sufixo casual.

$\begin{array}{ll}\begin{array}{l}\text { Formas longas em funçáo de O em Kaxararí } \\ \text { il awatşa pitu }\end{array} & \text { 'eu comi anta' } \\ \text { mil awatșa pitu } & \text { 'você comeu anta' } \\ \text { hal awatşa pitu } & \text { 'ele comeu anta' } \\ \text { lul awatşa pitu } & \text { 'nós comemos anta' } \\ \text { matul awatșa pitu } & \text { 'vocês comeram anta' } \\ \text { hatul awatşa pitu } & \text { 'eles comeram anta' }\end{array}$

Couto (2005) apresenta evidências de que há também alternância entre formas longas e curtas em Kaxararí, mas que, nessa língua, a alternância não é condicionada por sua função sintática. Nesse caso, consideramos que Kaxararí teria perdido a oposição entre formas longas e curtas e reanalisado as formas longas como a forma básica da palavra.

Esse autor observa ainda que, quando a última sílaba da palavra em Kaxararí possui $l \mathrm{em}$ margem direita de sílaba, sua variante curta mantém o lem final de palavra. Os seguintes exemplos são extraídos de Couto (op. cit.) e ilustram a alternância entre formas longas e curtas:

Exemplos de queda de última sílaba da palavra (COUTO, 2005):

$$
\begin{array}{lll}
\text { 20. }[\text { saba'ka }] & \sim \text { [sa'ba] } & \text { 'tipo de envireira' } \\
21 .[\text { awa'tsa }] & \sim\left[\mathrm{a}^{\prime} \mathrm{\prime} \mathrm{a} \emptyset\right] & \text { 'anta' }
\end{array}
$$

Exemplos de queda da vogal da última sílaba e manutenção de $l$ em final de palavra (COUTO, 2005).
22. [pana'la] [pa'nalø] 'açaí'
23. [kuna'l $\ll] \sim[\mathrm{ku}$ 'nalø] 'seringueira'

Exemplo de queda de consoante da última sílaba (COUTO, 2005).

24. [binu'ni] $\quad$ [bi'nuØi] 'buriti' 
Devido aos poucos dados disponíveis, não é possível saber da funcionalidade da alternância das formas longas e curtas. Náo se sabe, também, o grau de perda linguística pelos Kaxaraxí, nem se há variaçôes no uso mais ou menos conservador da língua, através das geraçôes. Entretanto, o que é relevante para a nossa discussão é que o Kaxararí também contribui com evidências para a hipótese de existência de consoantes finais de palavra no Protopáno.

\section{Consideraçóes finais}

No presente artigo, apresentamos uma proposta de reconstrução de consoantes finais em margem direitas de sílaba em Protopáno, com base em evidências de novas línguas antes não incluídas na reconstrução de Shell (1975 [1965]). Essa reconstrução considera a existência de uma alternância entre formas longas e curtas, na protolíngua, relacionada a sua função sintática, assim como ocorre na maior parte das línguas contemporâneas desta família. Discutiu-se, ainda, a natureza fonética dos segmentos reconstruídos, concluindo-se que, em Protopáno, também ocorreriam as consoantes ${ }^{*} \mathrm{~m},{ }^{*} \mathrm{n},{ }^{*} \mathrm{r},{ }^{*} \mathrm{r},{ }^{*} \mathrm{t}$, em margem direita de sílaba em final de palavra.

A proposta aqui apresentada leva ao reconhecimento de que o padrão $\mathrm{C}_{1} \mathrm{VC}_{2}$ em Protopáno poderia ter como $\mathrm{C}_{2}{ }^{*} \mathrm{~m},{ }^{*} \mathrm{n},{ }^{*} \mathrm{r},{ }^{*} \mathrm{r},{ }^{*} \mathrm{t}$ também no interior de palavras; no entanto, esse aspecto não pode ser explorado e deve ser abordado em futuras pesquisas. Da mesma forma, se estamos certos em afirmar que houve uma modificaçáo dos padróes silábicos do Protopáno às línguas contemporâneas, a existência do fenômeno de palavras terminadas em consoantes seria extensiva aos verbos.

Por fim, ressaltamos a necessidade de mais pesquisas descritivas sobre as línguas da família Páno, que considerem a alternância entre formas longas e curtas, seus condicionamentos e sua natureza morfofonológica. Seria também de especial interesse um estudo exaustivo sobre a interação entre esta alternância e os padróes acentuais, uma vez que as palavras que teriam uma consoante em final de palavra possuem um padrão acentual distinto do padrão geral em línguas como Shípibo e Marúbo.

\section{Referências}

ANONBY, S.; HOLBROOK, D. J. A survey of the languages of the Javari River Valley, Brazil. [S.l.]: SIL International, 2010. 
BARROS, L. G. A nasalizaçáo vocálica e fonologia introdutória à língua Katukína (Páno). 1987. Dissertação (Mestrado em Linguística), Universidade Estadual de Campinas, Campinas, SP, 1987.

CESARINO, P. N. Oniska: A poética da morte e do mundo entre os Marubo da Amazônia ocidental. 2008. 469f. Tese (Doutorado em Linguística), Universidade Federal do Rio de Janeiro, Rio de Janeiro, 2008.

CÓRDOBA, L.; VALENZUELA, P.; VILLAR, D. Las lenguas de los panos meridionales. 2011. mimeo.

COSTA, R. G. R. Aspectos da Fonologia Marubo (Pano): Uma visão nãolinear. 2000. Tese (Doutorado em Linguística), Universidade Federal do Rio de Janeiro, Rio de Janeiro, 2000.

COUTO, Alexandre. Ortografia Kaxarari: uma proposta. Porto Velho, 2005. (versão eletrônica)

EAKEN, L. Lecciones para el aprendizaje del idioma Yaminahua. Lima: Instituto Lingüístico de Verano, 2008.

FAUST, N.; LOOS, E. Gramática del Idioma Yaminahua. Peru: Instituto Lingüístico de Verano, 2002.

FERREIRA, R. V. Língua Matis (Pano): uma descrição gramatical. Tese (Doutorado em Linguística), Universidade Estadual de Campinas, Campinas, SP, 2005.

FLECK, D. W. A grammar of Matses. 2003. Tese (Doutorado em Linguística), Rice University, 2003.

GIRARD, V. J. Proto-Takanan phonology. Berkeley: UCPL 70, 1971.

HYDE, S. Diccionario Amahuaca. Yarinacocha: Ministerio de Educación; Instituto Lingüístico de Verano, 1980.

KENNEL JR., Gerald. Descriçáo fonêmica e gramatical do marubo. 1978. mimeo.

LOOS, E. La señal de transitividad del sustantivo en los idiomas pano. Serie Lingüística Peruana, n. 10, p. 133-184, 1978.

LORIOT, J.; LAURIAULT, E.; DAY, D. Diccionario Shipibo-Castellano. Yarinacocha, Pucallpa: Ministerio de Educación del Perú e Instituto Lingüístico de Verano, 1993. 
LOOS, E.; LOOS, B. Diccionario Capanahua Castellano. Yarinacocha, Pucallpa: Instituto Lingüístico de Verano, 1998.

MENDES, H. C. Os direcionais em Katukína. 1996. mimeo.

MNTB: Missão Novas Tribos do Brasil. Dados de campo. s/d. Acervo de Loos.

OLIVEIRA, S. C. S. de. Contribuiçóes para a Reconstruçáo do Protopáno. Tese (Doutorado em Linguística), Universidade de Brasília, UnB, Brasília, 2014.

PAULA, A. S. de. A língua dos índios Yawanawa do Acre. 2004. Tese (Doutorado em Linguística), Universidade Estadual de Campinas, Campinas, SP, 2004.

SPARING-CHÁVEZ, M. Aspects of Amahuaca Grammar: an endangered language of the Amazon Basin. 2012.

SHELL, O. A. Las lenguas pano y su reconstrucción. Lima: ILV/MEd, 1975.

VALENZUELA, P. M. Transitivity in Shipibo Konibo Grammar. 2003. Tese (Doutorado em Linguística), University of Oregon, Oregon, 2003.

VALENZUELA, P.; OLIVEIRA, S. Dados de pesquisa de campo. 2012. mimeo.

ZARIQUIEY, R. A grammar of Kashíbo-Kakataibo. 2011. Tese (Doutorado em Linguística), La Trobe University, Bundoora, Victoria, 2011.

ZINGG, P. Diccionario Chacobo-Castellano. La Paz: Ministerio de Educación, Cultura y Deportes y Confederación de Pueblos Indígenas Bolivia, 1998. 